\title{
NOTA PRELIMINAR SOBRE A COMPOSIÇÃO DO ZOUPLÂNCTON DO ESTUÁRIO DO RIO PARAÍBA DO NORTE,
} PB, BRASIL

\author{
GILBERTO ALVES PEKALA
}

Universidade Federal da Paraíba, João Pessoa, PB, Brasil

\section{SYNOPSIS}

Plankton samples were collected from 13 stations on the estuary of the river Paratiba do Norte $106^{\circ} 56^{\prime}$ to 070 09' Lat. S). Copepoda were the most abundant holoplanktonic organism and from the meroplankton the larvae of Cirripedia were the best represented in the interior the most abundant holoplanktonic organism and almost all the stations being always more abundant than Lamellibranchiae ones, mostly near the mouth of the channel. Decapoda larvae and eggs and larvae of fishes were also frequent. The spatial and temporal distribution of zooplankton will be studied in the future.

\section{Introdução}

O estudo da distribuição e o "standing-crop" do zoopláncton de estuários são de fundamental importáncia para uma compreensão científica acurada dos processos e mecanismos que atuam nesses ceossistemas. A distribuição e a composição do zooplâncton em estuários são afetadas pelos seguintes fatores: variaçōes em salinidado devida à maré: padrões de circulacão da água; gradientes ecológicos que se estabeiecem, sendo que o gradiente de salinidade é um dos mais importantes.

O estuário referente ao presente trabalho é formado, princi palmente, pelo rio Paraíba do Norte que apresenta um curso de 480 $\mathrm{km}$, sendo o mais extenso dos rios consequentes da Serra da Borborema Oriental Segundo Andrade (1959) a bacia hidrografica do Paraiba ocupa aproximadamente $32 \%$ da área territorial do Estado da Paraíba.

O estuário contém uma série de ilhas, tais como Portinho, Tiriri e Stuart formando vários canais, sendo que a ilha da Restinga, próxima da barra, divide o estuário em dois canais principais. Este complexo de ilhas, provavelmente, influiria no padrão de circulação do estuário, determinando correntezas de maior ou menor intensidade nestes canais. O estuário recebe, ainda, a contribuição dos rios Sanhauá, Mandacaru, Portinho, Ribeira e Guia. Formação de mangue é obeservada nas margens dos rios e nas ilhas.

Este trabalho teve como objetivo, um estudo preliminar sobre o levantamento e composição relativa da fauna planctônica ao longo do estuário.

\section{MATERIAL E MÉTODOS}

\section{Localização da área e das estações}

A região do estuário do rio Paraíba do Norte está compreendida entre as latitudes $06^{\circ} 56^{\prime} \mathrm{S}$ a $07^{\circ} 09^{\prime} \mathrm{S}$. As estações para a coleta do material, em número de 13 , foram escolhidas ao longo do estuário conforme mostra a Figura 1. de maneira a abranger todo n provável gradiente de salinidade, cujos valores variam de 6 a $30 \%$ na maré enchente e de 1 a $17 \%$ na maré vazante, respectivamente nas áreas onde se localizam as estaçōes I e VII (dados não publica(tos).

\section{Coleta do material}

O material foi coletado nos dias 12 e 13 de março de 1977 , durante a maré alta em algumas estações e em outras durante a maré baixa. Para obtenção das amostras de zooplâncton foi utilizada uma rede cônica de $200 \mu \mathrm{m}$ de abertura de malha, medindo $1 \mathrm{~m}$ de comprimento e $30 \mathrm{~cm}$ de diâmetro. Em cada estação, as amostras foram obtidas através de arrasto horizontal, durante 5 minutos com a velocidade do barco constante. O material foi fixado com formol a $4 \%$ neutralizado $\mathrm{e}$ a análise posterior do volume do plâncton foi feita através do método de decantação. A identificação dos organismos zooplanctônicos foi baseada em Trégouboff \& Rose (1957) e a contagem foi feita através de microscópio estereoscópico com aumento de 100 vezes Para obtenção do númern total de organismos em cada amostra, uma subamostragem de $2 \mathrm{cc}$ através de pipeta de "Stempel foi efetuada, sendo que o número obtido nessa subamostragem foi extrapolado para um volume previamente conhecido.

\section{Resultados e Discussões}

A r r igura 2 mostra o volume cio plâncton obtido nas diferentes estaçōes durante a maré al ta e maré baixa. A amostra que apresentou maior volume de pâncton $(78 \mathrm{cc})$ foi a estação I, coletada durante a maré alta sendo que o grupo da Medusae foi o responsável pelo alto valor do volume obtido, ocupando mais de $60 \%$ do volume total.
As amostras das estaçōes I a Vl, ıucalizada mais para o interior do estuário, apresentaram uma relação direta do volume do plâncton com o fluxo da maré, enquanto que, para as estaçōes VII a XIII, localizadas mais próximas ao canal de entrada de águas oceânicas, houve uma relação inversa. Isto pode ser explicado, possivelmente, pelo fato dos organismos serem carregados durante a maré al ta para o intenor do estuário, acarretando aumento do volume do pláncton nas estações localizadas nesta área em detrimento do volume do plâncton para as estações próximas à barra. Na maré vazante acontece o inverso pois os organismos são transportados em direção ao canal de saída havendo, conseqüentemente, aumento do volume do plâncton para as estações junto à barra e a diminuição nas estações localizadas no interior do estuário. Essa idéia pode ser ainda comprovada pela análise da composição qualitativa do zoonlâncton, demonstrando a ocorrência de certos organismos característicos de águas costeiras nas estações do interior.

A composição relativa do zooplâncton nas diferentes estações e os grupos analisados estão relacionados na Tabela I. Dos organismos holoplanctônicos analisados (Foraminifera, Medusae, Chaetognatha. Copepoda, Appendicularia e Dolliolida), os Copepoda foram sempre os mais abundantes, estando presentes em maior quantidade nas estacōes localizadas próximas à entrada da barra, contribuindu com uma média de $38,27 \%$ do total do zooplâncton, como pode ser observado na Figura 3.

Os Chaetognatha estiveram presentes em quase todas as estações, porém, uma representatividade maior em relação ao zooplâncton total foi observada nas estações X e XIII. Os Dolliolida estiveram presentes somente nas estaçōes próximas à entrada do canal enquanto que as Appendicularia apresentaram maior penetração para o interior do estuário. As Medusae ocorreram em quase todas as estaçōes, porém, numericamente não tiveram grande representação em relação ao zooplâncton total.

Os grupos dos Chaetognatha, Appendicularia e das Medusae segundo Paranaguá (1964) têm um papel bastante significativo na vida planctônica, pois a associação de espécies dos referidos grupos pode servir como indicadores de massas de água.

Quanto aos organismos meroplanctônicos, as larvas de Cirri. pedia tiveram grande representação nas estações localizadas mais para o interior do estuário contribuindo na maioria das amostras com mais de $70 \%$ do total do zooplâncton. Esta ocorrência está de acordo com as observacões de Tundisi (1970) cuja distribuição das larvas de Cirripedia esta relacionada com os movimentos de água doce na superfície associados com a contra corrente em direção às nascentes. É possível também que as larvas de Cirripedia sejam mais abundantes nas porções superiores do estuário devido a maior tolerância à salinidade apresentada por estes organismos.

Entre as larvas de Mollusca, véligers de Gastropoda foram mais abundantes, ocorrendo em quase todas as estações, enquanto que as véligers de Lamellibranchia ocorreram somente nas estaçōes próximas a entrada do canal onde a salinidade é mais alta. 1sto se confirma com os dados de Matsumura-Tundisi (1972) obtidos na região estuarina de Cananéia onde observou, para as véligers de Gastropoda, maior tolerância à diminuição de salinidade do que para as véligers de Lamellibranchia. O mesmo comportamento foi observado para as larvas de Polvchaeta

Outras larvas, Brachiura (caranguejo) e Macrura (camarão), como era de se esperar, estiveram presentes em grandes quantidades em todas as estações pois os estuários constrtuem o amoiente ideal para o desenvolvimento dessas larvas. Quanto aos ovos e larvas de peixes, foram frequientes, ocorrendo em quase todas as estações. sendo que os ovos foram mais abundantes nas estaçoes VII e X. Futuros estudos no estuário do rio Paraíba do Norte darão ênfase aos aspectos da distribuição espacial e temporal do zooplâncton em função dos gradientes horizontais e verticais de salinidade. 


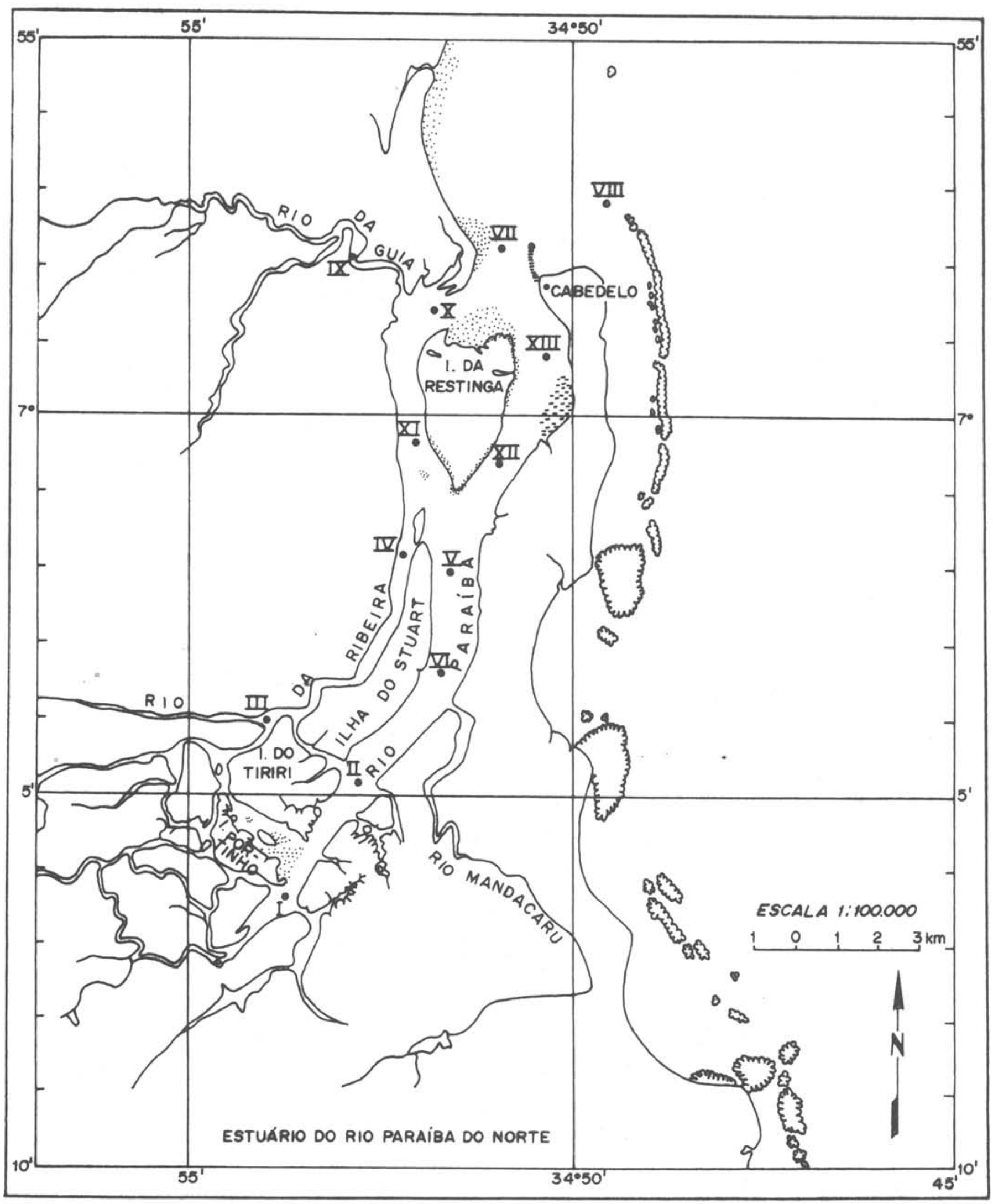

Fig. 1. Mapa mostrando a posição geográfica do estuário e a localização das estações de coleta. 
(w) $\exists \forall \forall W \quad \forall 0 \quad 7 \exists \wedge ! N$

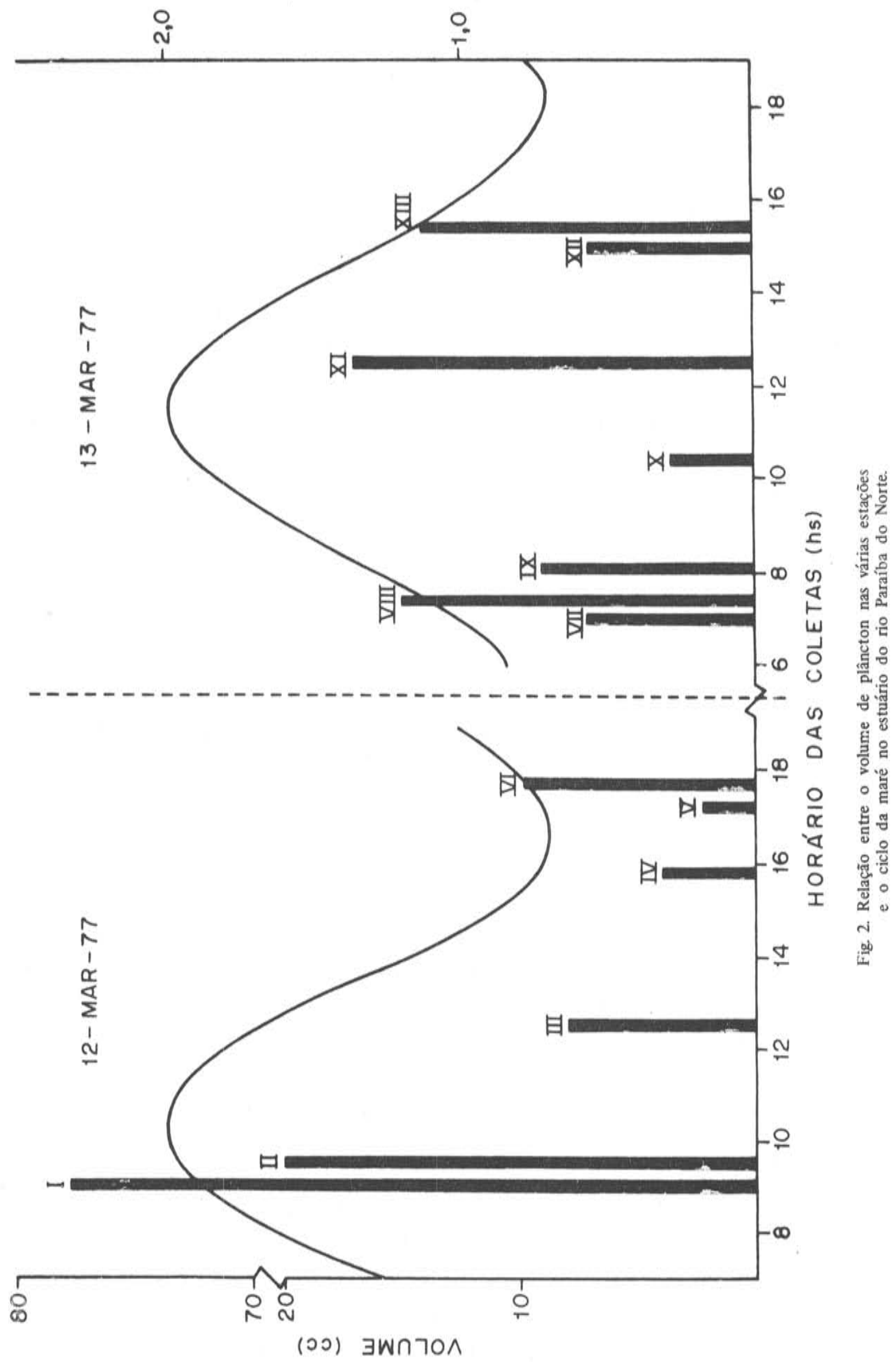




\begin{tabular}{|c|c|c|c|c|c|c|c|c|c|c|c|c|c|c|c|c|c|c|c|}
\hline 図 & & ถุ & $x$ & $\begin{array}{l}\tilde{a} \\
\dot{b} \\
\pm\end{array}$ & & & $\star$ & ஜ & & $\underset{\infty}{\infty}$ & $\approx$ & $\begin{array}{l}\text { ஜे } \\
\text { +. }\end{array}$ & $\begin{array}{l}\stackrel{\circ}{5} \\
\stackrel{+}{+}\end{array}$ & ఫి & $\begin{array}{c}\stackrel{n}{a} \\
\stackrel{0}{o}\end{array}$ & & $\begin{array}{r}\times 10 \\
\times \quad 2\end{array}$ & & $\begin{array}{l}\stackrel{\overbrace{}}{t} \\
\text { i }\end{array}$ \\
\hline 水 & $\underset{\infty}{\approx}$ & $\times$ & $\stackrel{n}{n}$ & $\begin{array}{l}\circ \\
\vdots \\
\\
\end{array}$ & & & $*$ & ஜ్ & & ฆู่ & & $\stackrel{8}{\circ}$ & $\begin{array}{l}n \\
\stackrel{n}{\infty} \\
\infty\end{array}$ & $\approx$ & 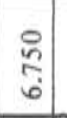 & & $\stackrel{m}{m} \Xi$ & ฟิ & 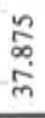 \\
\hline ヌ & & $\times$ & $\stackrel{n}{m}$ & $\begin{array}{l}\text { ठ্. } \\
\text { ठ․ }\end{array}$ & & & $x$ & है & & ఫ્ & $\underset{m}{n}$ & $\begin{array}{l}2 \\
0 \\
= \\
=\end{array}$ & $\begin{array}{l}\cong \\
⿱ 亠 䒑 \\
\vdots\end{array}$ & ఏ్ & $\begin{array}{l}2 \\
\vdots \\
= \\
=\end{array}$ & & $\beth \tilde{b}$ & & 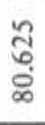 \\
\hline$x$ & & & $\stackrel{n}{m}$ & है & & $x$ & & ڤ్ & & ڤ્ & & ถู่ & $\underset{\substack{n \\
n}}{\stackrel{n}{n}}$ & 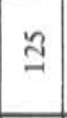 & $\stackrel{n}{\stackrel{n}{2}}$ & & 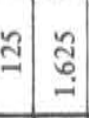 & & $\stackrel{2}{\stackrel{m}{g}}$ \\
\hline$\underline{\square}$ & & $\times$ & $x$ & ్ㅗ & & & * & $*$ & & 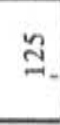 & & $\cong$ & $\begin{array}{l}\text { ठे } \\
\text { 广े } \\
\end{array}$ & $\star$ & ผ & & $\times$ ¿ & & 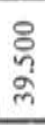 \\
\hline$\equiv$ & $\approx$ & $\cong$ & ڤి & 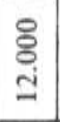 & ڤ્ & $\cong$ & 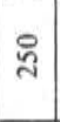 & : & สิ & $\stackrel{\text { ¿̊. }}{=}$ & $\underset{-}{\stackrel{0}{0}}$ & $\cong$ & ठ̊. & $\cong$ & \begin{tabular}{l}
$\stackrel{2}{\infty}$ \\
\hdashline \\
-1
\end{tabular} & & $\beth$ & ㅇ․․ & 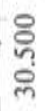 \\
\hline 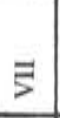 & $\stackrel{\beth}{\beth}$ & & & $\begin{array}{l}8 \\
6 \\
\vdots \\
-\end{array}$ & & & 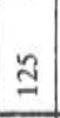 & & ஜ్ & ํ. & $\cong$ & ఫิ & $\begin{array}{l}n \\
\infty \\
\infty \\
\infty \\
\infty\end{array}$ & ๘ู & ถุ & & \begin{tabular}{l}
2 \\
\multirow{2}{*}{}
\end{tabular} & $\stackrel{n}{m}$ & $\stackrel{\text { m }}{\Xi}$ \\
\hline 5 & & $\cong$ & & $\stackrel{\circ}{\stackrel{2}{2}}$ & & & & & & ถุ & & & 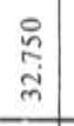 & & $\tilde{\zeta}$ & & & & $\begin{array}{l}8 \\
0 \\
5 \\
m \\
m\end{array}$ \\
\hline$>$ & & & $\times$ & $\stackrel{\infty}{\stackrel{\infty}{-}}$ & & & & & & ్ㅗㄱ & $\cong$ & 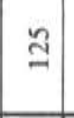 & $\begin{array}{l}\text { ڤ్ } \\
\text { ă }\end{array}$ & $*$ & 잉 & & $x$ & & 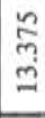 \\
\hline$\geq$ & & $x$ & & $\underset{i}{i}$ & & & & & & $\star$ & & & $\begin{array}{l}\text { ¿্் } \\
\text { m } \\
\text { m }\end{array}$ & $\frac{n}{m}$ & 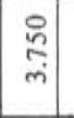 & & $\times \quad x$ & & 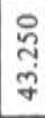 \\
\hline$\Xi$ & & $x$ & * & $\stackrel{2}{\leftrightarrows}$ & & & & & & 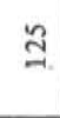 & & & $\begin{array}{l}\tilde{\approx} \\
\infty \\
\infty \\
\infty\end{array}$ & $\underset{\infty}{\check{\infty}}$ & 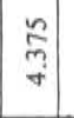 & & $\Xi \times$ & & $\begin{array}{l}\text { 号 } \\
\text { zे } \\
\text { y }\end{array}$ \\
\hline$=$ & & $x$ & $\beth$ & $\begin{array}{l}8 \\
\text { ¿ } \\
\end{array}$ & & & & & & & & $\times$ & $\begin{array}{l}\stackrel{2}{a} \\
\pm \\
\pm\end{array}$ & ส & $\begin{array}{l}\tilde{1} \\
\infty \\
\alpha \\
\end{array}$ & $\cong$ & $\times$ & & $\begin{array}{l}\stackrel{8}{5} \\
\frac{1}{2} \\
\end{array}$ \\
\hline- & & $\stackrel{n}{n}$ & $\times$ & $\begin{array}{l}8 \\
\stackrel{7}{+} \\
+\end{array}$ & & & & & & $\approx$ & & & $\begin{array}{l}2 \\
\frac{2}{3} \\
o \\
0\end{array}$ & $\begin{array}{l}n \\
\vdots \\
\infty \\
i \\
i\end{array}$ & \begin{tabular}{|l|} 
\\
$\vdots$ \\
$\infty$ \\
$-\infty$
\end{tabular} & & 이 & & 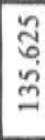 \\
\hline 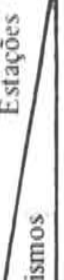 & 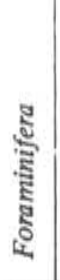 & ๕ั้ & 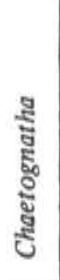 & 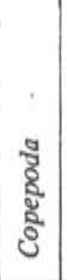 & 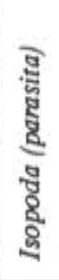 & 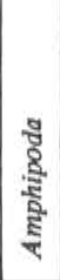 & 芯 & 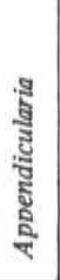 & 恋 & 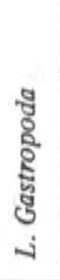 & 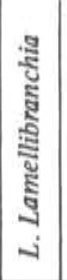 & 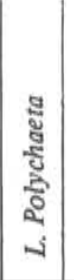 & 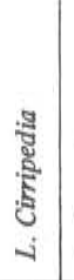 & 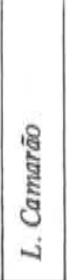 & 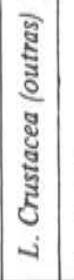 & 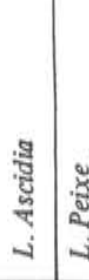 & 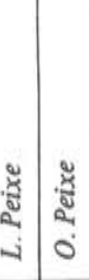 & 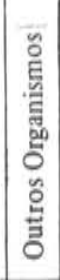 & 홍 \\
\hline
\end{tabular}



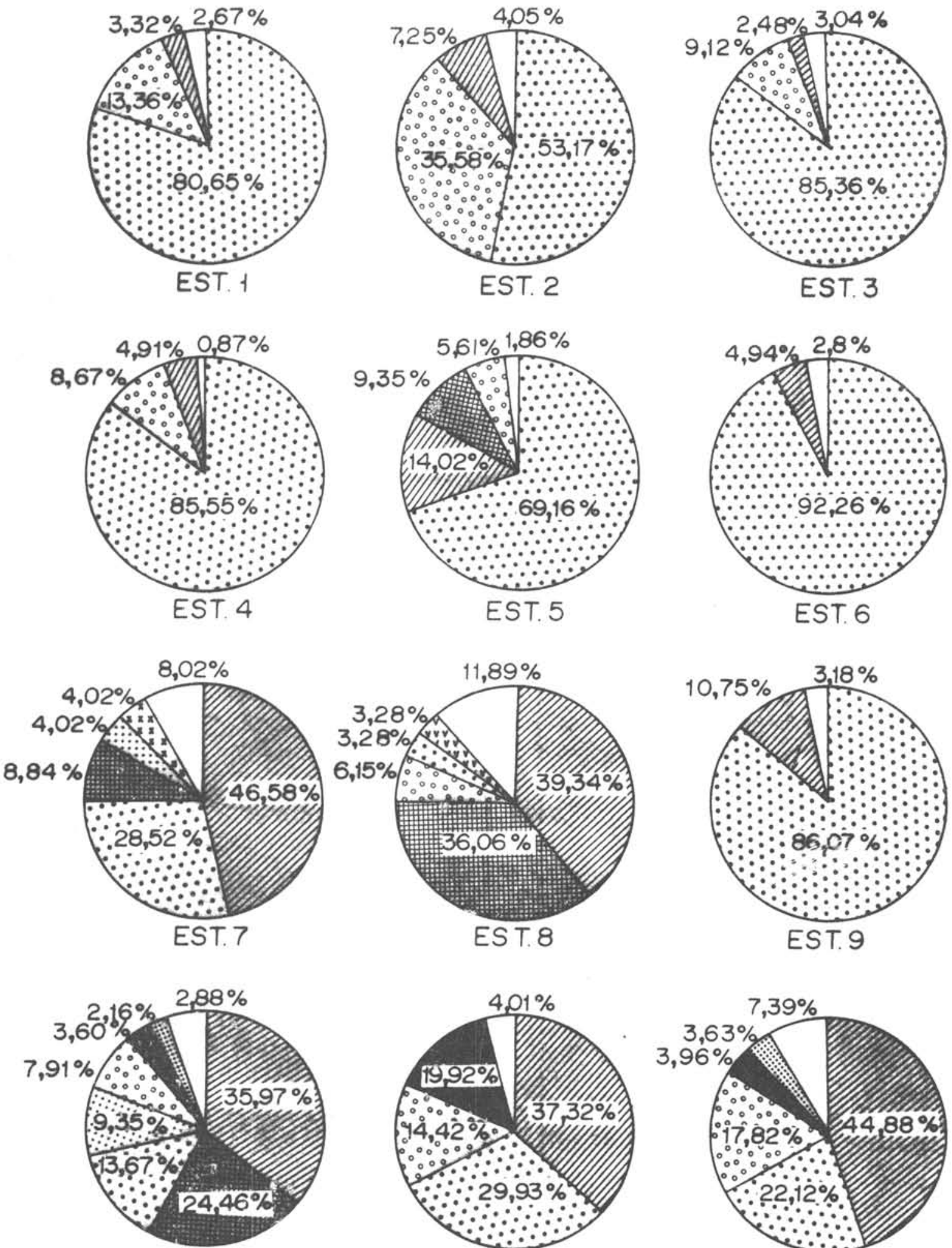

EST. 10
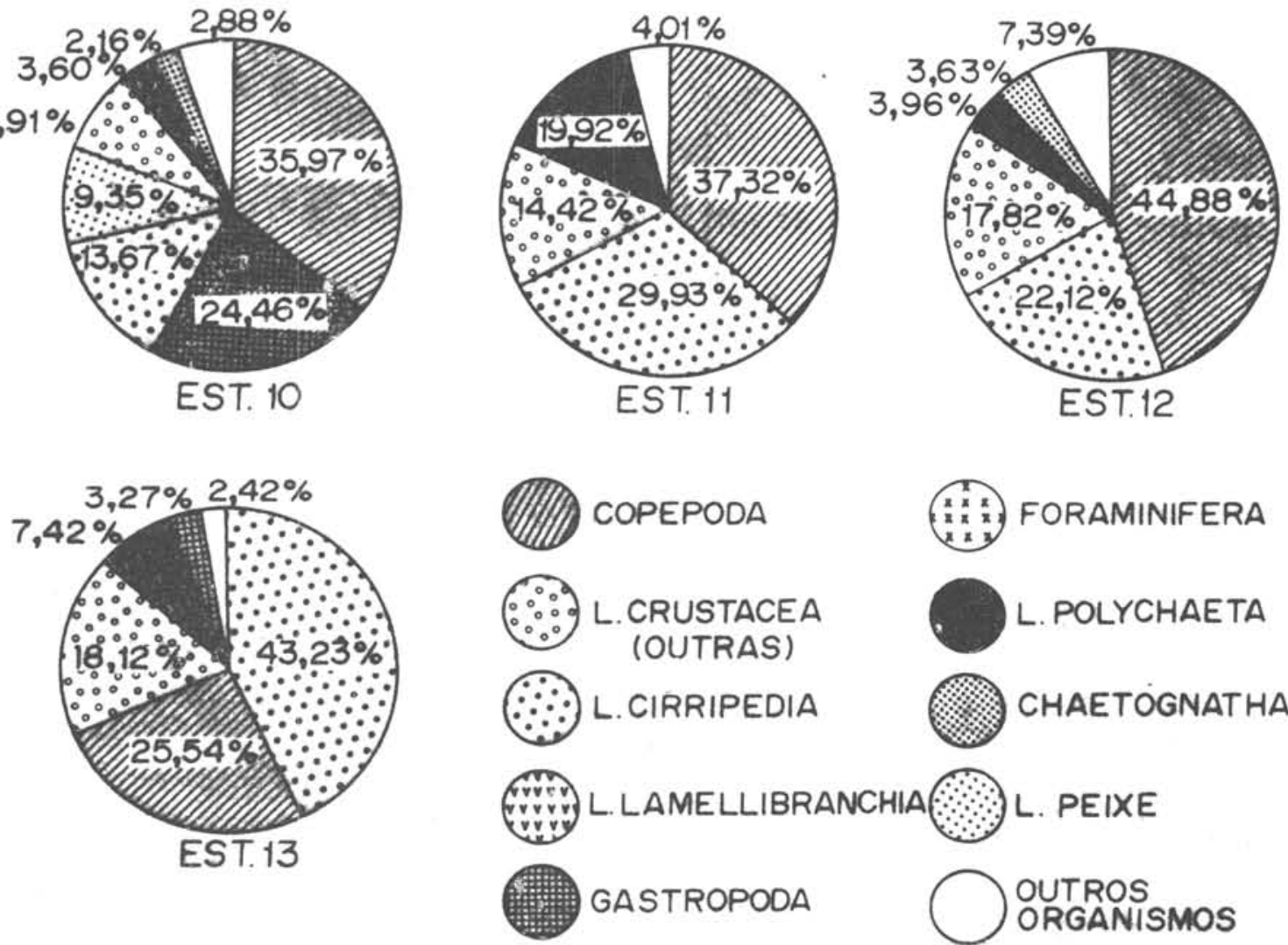

Fig. 3. Composição relativa do zooplâncton total e sua vanação nas diversas estações do estuário. 


\section{Agradecimentos}

Expresso os meus sinceros agradecimentos à Dra. T. Matsumura Tundisi e ao Dr. J.G. Tundisi do Departamento de Ciências Biológicas da Universidade Federal de São Carlos, pelas críticas e sugestōes ao manuscrito.

Agradeço ao Laboratório de Plâncton do Departamento de Oceanografia da Universidade Federal de Pernambuco pelas facilidades concedidas para a análise do material e à Dra. M. N. Paranagua pelo auxílio prestado na identificação dos organismos planctônicos.

\section{Bibliografia}

ANDRADE, G.O. 1959. Os rios-do-açúcar do Nordeste Oriental. III $\mathrm{O}$ rio Paraíba do Norte. Recife, Instituto Joaquim Nabuco de Pesquisas Sociais, $155 \mathrm{p}$.
MATSUMURA-TUNDISI, T. 1972. Aspectos ecológicos do zooplâncton da região lagunar de Cananéia com especial referência aos Copepoda (Crustacea). Tese de Doutorado. Universidade de São Paulo, Institu to de Biociências.

PARANAGUÁ, M.N. 1964. Sobre o pläncton da região compreendida entre $3^{\circ}$ Lat. S e $13^{\circ}$ Lat. $S$ ao largo do Brasil. Trabhs Inst. oceanogr. Univ. fed. Pernambuco, $5 / 6: 125-139$

TRÉGOUBOFF, G. \& ROSE, M. 1957, Manuel de planctonotogie méditerranénne. Paris, Centre National de la Recherche Scientifique, $2 \mathrm{v}$

TUNDISI, J.G. 1970. O plâncton estuarıno. Contrções Inst. oceanogr. Univ, S Paulo, sér. Oceanogr. biol., (19) :1-22. 